\title{
Seizures and Brain Injury in Neonatal Rats Induced by 1S,3R-ACPD, a Metabotropic Glutamate Receptor Agonist
}

\author{
John W. McDonald, ${ }^{1}$ Andrew S. Fix, ${ }^{2}$ Joseph P. Tizzano, ${ }^{2}$ and Darryle D. Schoepp ${ }^{1}$ \\ ${ }^{1}$ CNS Research Division and ${ }^{2}$ Toxicology Research Laboratories, Lilly Research Laboratories, Eli Lilly and Company, \\ Indianapolis, Indiana 46285
}

\begin{abstract}
The role of metabotropic excitatory amino acid receptors in seizures and brain injury was examined using the selective metabotropic agonist 1S,3R-ACPD [(1S,3R)-1-aminocyclopentane-1-3-dicarboxylic acid $\mid$ in 7-d-old neonatal rats. Systemic administration of 1S,3R-ACPD produced dose-dependent convulsions $\left(E D_{50}=16 \mathrm{mg} / \mathrm{kg}\right.$, i.p.) that were stereoselective for the active metabotropic ACPD isomer, since $1 R, 3 S-A C P D$ was less potent $\left(E D_{50}=93 \mathrm{mg} / \mathrm{kg}\right.$, i.p.). $1 S, 3 R$-ACPD-induced seizures were antagonized by systemic administration of dantrolene, an inhibitor of intracellular calcium mobilization, but not by the ionotropic glutamate antagonists MK-801 or GYKI-52466. As indexed by hemispheric brain weight differences $5 \mathrm{~d}$ postinjection, unilateral intrastriatal injection of 1S,3R-ACPD (0.1-2.0 $\mu \mathrm{mol} /$ $\mu l)$, but not $1 R, 3 S-A C P D$, produced dose-dependent brain injury (maximal effect of $3.4 \pm 0.5 \%$ damage). $15,3 R-A C P D$ brain injury occurred in the absence of prominent behavioral convulsions. Histologic and ultrastructural examination of 1S,3R-ACPD-injected rat brains revealed swelling and degeneration of select neurons at $4 \mathrm{hr}$ postinjection, but little evidence of injured neurons 5 d later. 1S,3R-ACPD-mediated brain injury was not attenuated by systemic administration of the NMDA antagonist MK-801 or the AMPA antagonist GYKI-52466. However, cointrastriatal injection of dantrolene reduced the severity of $15,3 R$-ACPD injury by $88 \pm 7 \%$. These studies indicate that seizures and neuronal injury can be elicited by the selective activation of metabotropic glutamate receptors in perinatal rats, and these effects of $1 S, 3 R$ ACPD involve the mobilization of intracellular calcium stores.

[Key words: 1S,3R-ACPD (1-amino-cyclopentane-1,3-dicarboxylic acid), glutamate, brain injury, metabotropic glutamate receptors, dantrolene, excitatory amino acid]
\end{abstract}

Based on biochemical, electrophysiological, and molecular studies, excitatory amino acid receptors have been broadly classified as ionotropic (ion channel linked) or metabotropic (G-protein linked). Ionotropic receptors are subclassified into NMDA, $\alpha$-amino-3-hydroxy-5-methylisoxazole-4-propionic acid (AMPA), and kainate receptors based on their preferential agonists (Monaghan et al., 1989). More recently, metabotropic or guanine nucleotide binding protein-linked excitatory amino acid

Received Jan. 22, 1993; revised Apr. 19, 1993; accepted Apr. 29, 1993.

Correspondence should be addressed to Darryle D. Schoepp, Ph.D., CNS Research, MC907, DC 0815, Lilly Research Laboratories, Eli Lilly and Company, Indianapolis, IN 46285.

Copyright (C) 1993 Society for Neuroscience $0270-6474 / 93 / 134445-11 \$ 05.00 / 0$ receptors, which are coupled to phosphoinositide hydrolysis or modulation of cAMP metabolism, have been characterized (Sladeczek et al., 1985; Nicoletti et al., 1986a; Pearce et al., 1986; Sugiyama et al., 1987; Schoepp and Johnson, 1988; Schoepp et al., 1992b). Nonselective agonists of metabotropic receptors include glutamate, ibotenate, and quisqualate (Schoepp et al., 1990a). Recently, more selective metabotropic agonists have been developed, including ( \pm trans-ACPD [( \pm -trans-1-aminocyclopentane-1,3-dicarboxylic acid (Palmer et al., 1989; Desai and Conn, 1990)]. Trans-ACPD is a racemic mixture of $1 S, 3 R$ and $1 R, 3 S$ stereoisomers. $1 S, 3 R$-ACPD is the active isomer of trans-ACPD and is currently the most selective and potent metabotropic agonist available (Schoepp et al., 1991b). In contrast, $1 R, 3 S$-ACPD is a relatively inactive agonist at metabotropic glutamate receptors that are linked to phosphoinositide hydrolysis or modulation of adenylate cyclase activity (Irving et al., 1990; Schoepp et al., 1991b). The second messenger responses to metabotropic agonists are not antagonized by selective ionotropic antagonists (Nicoletti et al., 1986a; Schoepp and Johnson, 1988; Schoepp et al., 1992a).

In contrast to ionotropic-type excitatory amino acid receptors, the physiologic and pathologic roles of metabotropic receptors are just beginning to emerge because of the availability of a selective agonist. Recent reports indicate that $1 S, 3 R$-ACPD can produce brain injury. Intrastriatal injection of subtoxic doses of $1 S, 3 R$-ACPD potentiates NMDA- but not AMPA-mediated brain injury in neonatal rats (McDonald and Schoepp, 1992). Stereotaxic intrahippocampal injection of higher doses of $1 S, 3 R$ ACPD produces delayed seizures and prominent neuronal injury in adult rats (Sacaan and Schoepp, 1992).

The developing CNS provides a unique model to assess metabotropic excitatory amino acid receptor function and interactions with ionotropic receptors, since metabotropic responses (i.e., phosphoinositide hydrolysis) are transiently enhanced during the early postnatal period (McDonald and Johnston, 1990; Schoepp and Hillman, 1990; Schoepp et al., 1990a). Furthermore, chemosensitivity and excitotoxicity of NMDA and AMPA receptors are also markedly increased during this same period (McDonald et al., 1988; Ikonomidou et al., 1989; McDonald and Johnston, 1990). In this study we further examined the ccllular mechanisms and pharmacology of scizures and ncuronal injury associated with the selective activation of $1 S, 3 R$-ACPDsensitive metabotropic glutamate receptors in neonatal rats.

\section{Materials and Methods}

Animals. Postnatal day (PND) 7 male and female Sprague-Dawley albino rats were used in all experiments (Charles River Laboratories, Inc., 


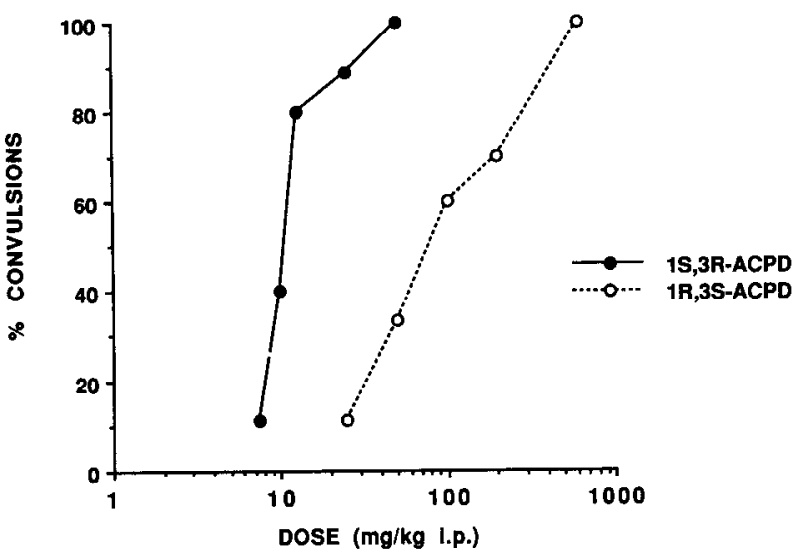

Figure 1. Comparison of dose-effect curves for convulsions produced by intraperitoneal administration of either $1 S, 3 R$-ACPD or $1 R, 3 S$ ACPD. PND 7 rats were observed for behavioral signs of motor seizures over a $30 \mathrm{~min}$ period following intraperitoneal injection of ACPD isomers $(0.05 \mathrm{ml}$ in PBS, $\mathrm{pH} 7.4)$. Ten animals were tested at each dose. The vertical axis represents the percentage of animals that exhibited motor convulsions at a particular dose of ACPD.

Wilmington, MA). Litters were culled to 12 pups and animals were maintained on a 12:12 hr light: dark cycle.

Convulsions in neonatal rats. Evaluation of convulsant behavior in PND 7 rats was carried out as described previously (Schoepp et al., $1990 \mathrm{~b})$. Littermate pairs received one of five doses of either $1 S, 3 R$ ACPD $(7.5-100 \mathrm{mg} / \mathrm{kg})$ or $1 R, 3 S$-ACPD $(25-500 \mathrm{mg} / \mathrm{kg})$. Five to 10 animals were tested at each dose. All compounds were administered intraperitoneally in phosphate-buffered saline or sterile water $(10 \mathrm{ml}$ $\mathrm{vol} / \mathrm{kg}$ body weight). Following the injection of agonists, animals were placed in warmed $\left(36^{\circ} \mathrm{C}\right)$ Plexiglas observation chambers, evaluated for $30 \mathrm{~min}$, and scored for the presence or absence of tonic or clonic motor movements. In some experiments, antagonists were administered 30 min prior to $1 S, 3 R$-ACPD. Data were expressed as percentage of animals exhibiting convulsions or as number of animals convulsed/number of animals tested. The convulsant dose of the agonist in $50 \%$ of the animals tested $\left(E D_{50}\right)$ was calculated using the median-effect plot of Chou and Talalay (1983).

Brain injury in neonatal rats. The pharmacology of ACPD-induced injury was assessed in a well-characterized in vivo neonatal rat model (McDonald et al., 1989). Briefly, PND 7 rats received unilateral intrastriatal or hippocampal stereotaxic injections of $1 S, 3 R$-ACPD (or $1 R, 3 S$-ACPD). Injection coordinates relative to bregma were (1) striatum = AP $0 \mathrm{~mm}$, ML $2.5 \mathrm{~mm}, \mathrm{~V} 4.0 \mathrm{~mm}$ from the dura matter and (2) hippocampus - AP $3 \mathrm{~mm}$, ML $2 \mathrm{~mm}, \mathrm{~V} 2.5 \mathrm{~mm}$ from the dura matter. Injections were completed over a 2 min period using a 26 gauge Hamilton syringe. Drugs for intracerebral injection were dissolved in $0.01 \mathrm{~m}$ Tris ( $\mathrm{pH} 7.4$ ) and injection volumes were either 0.5 or $1.0 \mu \mathrm{l}$ depending on coinjection of other drugs: the severity of brain injury resulting from injection of $1000 \mathrm{nmol}$ of $1 S, 3 R$-ACPD did not differ between $0.5 \mu 1$ or $1.0 \mu$ injection volumes. Administration of potentially neuroprotective drugs was performed either by cointrastriatal injection or intraperitoneal administration $(10 \mathrm{ml} \mathrm{vol} / \mathrm{kg}$ body weight $)$. Eight to 10 animals per group were used in the neuroprotective studies. Body temperature was maintained at normothermia $\left(36^{\circ} \mathrm{C}\right.$ surface temperature) using a hovabator incubator.

Five days after the injection, the severity of brain injury was assessed by loss of injected $(I)$ cerebral hemisphere weight relative to the contralatcral $(C)$ sidc using the formula \% damage $=100 \cdot(C-I) / C$. In this model reductions of hemisphere weights are highly correlated with regional reductions in ChAT activity and cross-sectional area measurements (McDonald et al., 1989). Statistical analysis consisted of Student's $t$ test for independent values.

Morphologic examination. Morphologic examinations were made by light and electron microscopy in PND 7 rats that received injections of vehicle and $1 S, 3 R$-ACPD into the striatum or hippocampus (as described above). At $4 \mathrm{hr}$ or $5 \mathrm{~d}$ postinjection, rats were deeply anesthetized with methoxyflurane and perfused through the left ventricle with a heparinized saline flush followed by a fixative containing $4 \%$ parafor-
Table 1. Effect of excitatory amino acid receptor antagonists on $1 S, 3 R$-ACPD-induced seizures in neonatal rats

\begin{tabular}{llc} 
Pretreatment & Agonist & $\begin{array}{c}\text { \#Convulsed } \\
\text { \# tested }\end{array}$ \\
\hline Vehicle & Vehicle & $0 / 10$ \\
Vehicle & $1 S, 3 R$-ACPD $(40 \mathrm{mg} / \mathrm{kg})$ & $10 / 10$ \\
MK-801 $(1 \mathrm{mg} / \mathrm{kg})$ & $1 S, 3 R$-ACPD $(40 \mathrm{mg} / \mathrm{kg})$ & $9 / 10$ \\
GYKI-52466 $(20 \mathrm{mg} / \mathrm{kg})$ & $1 S, 3 R$-ACPD $(40 \mathrm{mg} / \mathrm{kg})$ & $9 / 10$ \\
Dantrolene $(500 \mathrm{mg} / \mathrm{kg})$ & $1 S, 3 R$-ACPD $(40 \mathrm{mg} / \mathrm{kg})$ & $0 / 10^{a}$
\end{tabular}

Antagonists or sterile water (vehicle) were given by the intraperitoneal route 30 min prior to intraperitoneal injection of $1 S, 3 R$-ACPD or its water vehicle. Animals were observed for behaviors for $30 \mathrm{~min}$ before and an additional $30 \mathrm{~min}$ after $1 S, 3 R$-ACPD injection.

${ }^{a}$ Minimally effective protective dose ( $\geq 50 \%$ protection).

maldehyde $/ 1.4 \%$ glutaraldehyde. Examinations made at $5 \mathrm{~d}$ postinjection were limited to rats that received intrastriatal injections and were only by light microscopy. Fixed brains were removed immediately after perfusion and stored in cold fixative until trimming with a rat brain matrix (Activational Systems Inc., Warren, MI). For light microscopy, trimmed slices were processed by graded ethanol dehydration, embedded in paraffin blocks, sectioned at $4 \mu \mathrm{m}$, stained with hematoxylin and eosin (HE), and examined by light microscopy. For transmission electron microscopy, select slices were secondarily fixed in a solution of cold $2.0 \%$ paraformaldehyde $/ 2.5 \%$ glutaraldehyde, rinsed with $0.1 \mathrm{M}$ sodium cacodylate buffer (pH 7.2), postfixed in aqueous $1 \%$ osmium tetroxide $/ 1.5 \%$ potassium ferrocyanide on ice, serially dehydrated in ethanols, and embedded in epoxy resin. Epoxy blocks were sectioned at $1 \mu \mathrm{m}$, stained with toluidine blue, and examined by light microscopy. After identification of select target areas, ultrathin sections were cut with a diamond knife, stained with uranyl acetate and Sato's lead citrate, and examined with a Philips 410LS transmission electron microscope.

Materials. MK-801 [(+)-5-methyl-10,11-dihydro-5H-dibenzo[a,d]cyclohepten-5,10-imine maleate] was a gift from Dr. P. Anderson (Merck, Sharp and Dohme, West Point, PA). $1 S, 3 R$-ACPD $[(1 S, 3 R)-$ 1 -amino-cyclopentane-1,3-dicarboxylic acid] was obtained from Tocris Neuramin (Essex, UK). Dantrolene was purchased from Sigma Chemical Company (St. Louis, MO). 1R,3S-ACPD was prepared as described by Schoepp et al. (1991b). GYKI-5246 was from the Institute for Drug Research (Budapest, Hungary).

\section{Results}

The convulsive effects of systemic administration of $1 S, 3 R$ ACPD and $1 R, 3 S$-ACPD were compared in PND 7 rats. Both ACPD isomers produced dose-related behavioral seizures (Fig. 1). $1 S, 3 R$-ACPD was approximately six times more potent than $1 R, 3 S$-ACPD, with $E_{50}$ values of $16 \mathrm{mg} / \mathrm{kg}$ and $93 \mathrm{mg} / \mathrm{kg}$, respectively. The pattern of behavioral seizures produced by either isomer could not be dissociated. Both $1 S, 3 R-A C P D$ and $1 R, 3 S$-ACPD produced exaggeration of naturally occurring myoclonus, followed by lordosis and rear wagging, and then repetitive tonic-clonic limb extension. The pharmacology of $1 S, 3 R$-ACPD-induced seizures was examined by pretreating animals with dantrolene, an inhibitor of intracellular calcium mobilization, the NMDA receptor antagonist MK-801, and the AMPA receptor antagonist GYKI-52466. At the doses used, each of these antagonists produced modest to moderate sedation as indexed by ataxic motor movements. However, among these agents, dantrolene was found to prevent selectively and completely the $1 S, 3 R$-ACPD-induced seizures (Table 1).

In contrast to systemic administration, intrastriatal injection of $1 S, 3 R$-ACPD produced few signs of clonic convulsions; intermittent tonic limb extension was occasionally observed. However, these intracerebral doses of $1 S, 3 R$-ACPD produced brain weight disparities indicative of brain injury. The quan- 
titative dose-effects of $1 S, 3 R$-ACPD and $1 R, 3 S$-ACPD are illustrated in Figure 2. When examined $5 \mathrm{~d}$ after unilateral intrastriatal injection on PND 7, $1 S, 3 R$-ACPD produced a dose-related reduction in the weight of the injected cerebral hemisphere. The lowest significant dose of $1 S, 3 R$-ACPD producing injury was $500 \mathrm{nmol}$, and maximal effect $(3.4 \pm 0.5 \%$ damage) was observed at the $2000 \mathrm{nmol}$ dose. The solubility limit for $1 S, 3 R$-ACPD did not allow injection of higher doses. In contrast to the effects of $1 S, 3 R-\mathrm{ACPD}$, the $1 R, 3 S$-ACPD isomer did not produce significant brain injury at doses up to $1000 \mathrm{nmol}$ as assessed by hemisphere weight disparities.

Light microscopy revealed injury to selective neurons in both striatum and hippocampus at $4 \mathrm{hr}$ postinjection in all rats that received $1 S, 3 R$-ACPD $(1000 \mathrm{nmol})$ injections. When compared to normal control neurons from similar regions, injured neurons had clear, swollen perinuclear cytoplasm containing remnant membranous debris. In addition, nuclei were slightly shrunken and pale in affected neurons (Figs. 3, 4). Injury was of similar character in neurons from both striatum and hippocampus, was not detected in glial and ependymal cells, and was consistent from animal to animal.

In striatum, injured neurons were scattered throughout the caudate-putamen near the injection site and were frequently located adjacent to morphologically normal neurons (Fig. 3). Injured neurons with a similar morphologic appearance were also evident in other brain regions ipsilateral to the injection site, including layers $3,4,5$, and 6 of cingulate, parietal, insular, and piriform cortex, and lateral septal nucleus. In the septum, neuronal injury was more pronounced than in affected cortical areas, where injury was rather scattered. Injured neurons contralateral to the injection site were observed only in the cingulate cortex and lateral septal nucleus. Brain sections evaluated by light microscopy $5 \mathrm{~d}$ postinjection contained no morphologic abnormalities.

In hippocampus, injured neurons were evident throughout the polymorphic, pyramidal, and molecular layers of hippocampal regions CA1-CA4 (Fig. 4). Based on qualitative light microscopy, susceptibility to the effects of $1 S, 3 R$-ACPD appeared to be greatest in the molecular and polymorphic layers. In these two layers, a greater percentage of neurons were injured than in the more dense pyramidal cell layer, where only occasional neurons were injured. Injured neurons were also evident in the dentate gyrus, but not to the extent as in the hippocampus. Other brain regions contained similarly appearing injured neurons ipsilateral to the injection site, including layers $3,4,5$, and 6 of retrosplenial, frontal, parietal, and perirhinal cortex, dorsal thalamic regions, and amygdala. Neuronal injury was not detected in any contralateral brain regions examined. Evaluations were not made at $5 \mathrm{~d}$ postinjection in rats that received hippocampal injections.

Transmission electron microscopic examination at $4 \mathrm{hr}$ postinjection of rats that received intrastriatal injections of $1 S, 3 R$ ACPD revealed severe disruption of normal cytoplasmic organelles in injured neurons (Fig. 5). The selectivity of injury noted by light microscopy was confirmed with electron microscopy, as injured neurons were frequently adjacent to neurons that lacked morphologic alterations. In contrast to normal control neurons from the same region, injured neurons had swollen, electron-lucent cytoplasm containing remnant organelles and membranous debris. Injured neurons also had degranulation of endoplasmic reticulum, scattered ribosomes, disrupted Golgi apparatus, swollen mitochondria with cristolysis,

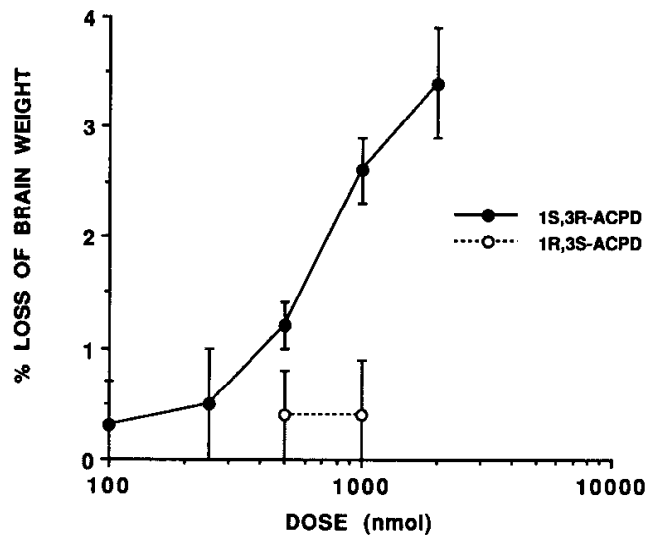

Figure 2. Dose-effects of unilateral intrastriatal injection of $1 S, 3 R$ ACPD or $1 R, 3 S$-ACPD on brain injury in the neonatal rat. Compounds were dissolved in 0.5 or $1.0 \mu \mathrm{l}$ of $0.01 \mathrm{M}$ Tris (pII 7.4) and were injected into the right striatum of ether-anesthetized PND 7 rats. The severity of brain injury was assessed 5 d later, on PND 12, by comparison of cerebral hemisphere weight disparities. Data are presented as percentage loss brain weight (mean $\pm \mathrm{SEM}, n=5-10$ per dose) using the formula $100 \cdot(C-I) / C$, which represents the reduction in the weight of the injected $(I)$ hemisphere relative to the contralateral $(C)$ hemisphere.

and large blebs at the plasmalemma. Dissolution and clearing of cytoplasmic particulate material were evident in some cells. Although some nuclei were slightly swollen and pale, condensation and margination of chromatin, loss of normal nucleolar organization, and mild nuclear membrane blebbing were characteristic. Slight axonal and dendritic swelling were evident in the neuropil.

The pharmacologic specificity of $1 S, 3 R$-ACPD-induced brain injury was examined by assessing the neuroprotective pharmacology of selective excitatory amino acid antagonists. Systemic administration of either the AMPA antagonist GYKI$52466(20 \mathrm{mg} / \mathrm{kg}$ twice, concurrent and $1 \mathrm{hr}$ after intrastriatal injection) or the NMDA antagonist MK-801 $(1 \mathrm{mg} / \mathrm{kg})$ did not attenuate the severity of injury produced by intrastriatal injection of $1000 \mathrm{nmol}$ of $1 S, 3 R$-ACPD. In contrast, cointrastriatal injection of dantrolene reduced $1 S, 3 R$-ACPD injury by $88 \pm$ 7\% (Fig. 6).

\section{Discussion}

Little information regarding the pathophysiologic roles of metabotropic excitatory amino acid receptors in brain injury is available (McDonald and Johnston, 1990; Schoepp et al., 1990a). This largely reflects the paucity of sclcctive compounds for metabotropic receptors. Using neonatal rats, we have characterized the in vivo pharmacology of convulsions and brain injury induced by the selective metabotropic agonist $1 S, 3 R$-ACPD. When administered systemically, $1 S, 3 R$-ACPD was six times more potent as a convulsant than $1 R, 3 S$-ACPD. In previous work, intrastriatal injection of a nontoxic dose of $1 S, 3 R$-ACPD (250 nmol) was found to potentiate NMDA toxicity in the neonatal rat (McDonald and Schoepp, 1992). In this study, higher doses of $1 S, 3 R$-ACPD were examined for direct neurotoxicity. We found that $1 S, 3 R$-ACPD ( $\geq 500 \mathrm{nmol}$ ) produced dose-dependent brain injury when injected intrastriatally, as indexed by hemispheric brain weight disparities $5 \mathrm{~d}$ postinjection. Like convulsions, this effect of $1 S, 3 R$-ACPD was also stereoselective, since $1 R, 3 S$-ACPD produced no significant brain weight losses at similar doses. 
The ACPD isomer potency differences observed for convulsions and neurotoxicity in this study agree with observations in neonatal rat brain slice assays measuring receptor transduction events such as stimulation of phosphoinositide hydrolysis (Schoepp et al., 1991b). In this context, 1S,3R-ACPD is at least 30 times more potent in activating metabotropic receptors compared to its affinity for NMDA receptors, and it has little affinity for AMPA or kainate receptors (Schoepp et al., 1991b; Sacaan and Schoepp, 1992). Thus, the stereoselectivity of effects in this study suggests that the convulsant and neurotoxic effects of $1 S, 3 R$-ACPD are likely due to the selective activation of metabotropic glutamate receptors in the neonatal rat brain.

Histological examination of $1 S, 3 R$-ACPD-injected rat brains confirmed the neurotoxic effects of this compound. This neuronal injury was characterized by select populations of swollen neurons with shrunken nuclei at $4 \mathrm{hr}$ postinjection, but no injured neurons could be found $5 \mathrm{~d}$ later. These data indicate that, similar to lesions produced by ionotropic glutamate agonists (McDonald et al., 1989), the brain weight disparities produced by $1 S, 3 R$-ACPD likely reflect earlier selective loss of neurons that manifests as reduced tissue volume $5 \mathrm{~d}$ later. However, $1 S, 3 R$-ACPD injury differs qualitatively from the injury produced by direct injection of ionotropic glutamate receptor agonists such as NMDA. For example, in the neonatal rat and mouse species NMDA produces acute changes in neurons that characteristically include edematous cell swelling, nuclear pyknosis, dark cell degeneration, and massive distention of dendrosomal regions that spares axons (Olney, 1978; Ikonomidou et al., 1989). Also evident in excitotoxic damage following NMDA are acute changes in glial and ependymal cells (Olney, 1971). In the present study, acute changes at 4 hr postinjection were limited to selective neuronal swelling without evidence of dark cell degeneration or dendrosomal involvement typical of excitotoxic damage. In addition, the lack of visible damage to glial or ependymal cells provides further support for the qualitative difference between $1 S, 3 R$-ACPD injury and excitotoxic injury typical of ionotropic glutamate receptor agonists.

Two previous studies using trans-ACPD suggested that metabotropic receptor activation did not produce neuronal injury in neocortical cultures (Koh et al., 1991) or when injected intrastriatally in rats (Schoepp et al., 1991a). These observations may be peculiar to the use of $( \pm)$ trans-ACPD. $( \pm)$ Trans-ACPD is a racemic mixture of $1 S, 3 R$-ACPD and $1 R, 3 S$-ACPD. $1 S, 3 R$ ACPD is the active agonist while $1 R, 3 S$-ACPD is a weak partial agonist of metabotropic-stimulated phosphoinositide hydrolysis (Schoepp et al., 1991b), which can attenuate receptor activation by the full agonist $1 S, 3 R$-ACPD (Schoepp et al., 1992b). This apparent antagonist or partial agonist effect of $1 R, 3 S$-ACPD has been observed when measuring $1 S, 3 R$-ACPD-induced contralateral turning, a behavioral consequence of activating striatal metabotropic glutamate receptors with $1 S, 3 R$-ACPD in the adult rat (Schoepp et al., 1992b). Thus, $1 R, 3 S$-ACPD may negate a portion of the agonist (toxic) properties of $1 S, 3 R$-ACPD by acting as a functional antagonist. Furthermore, in our previous study with $( \pm)$ trans-ACPD significant levels of brain injury were achieved with only the highest injectable intrastriatal dose of trans-ACPD (1200 nmol) in PND 7 rats (Schoepp et al., 1991a). This degree of injury reflects only the amount of injury expected to be induced by $600 \mathrm{nmol}$ of the active $1 S, 3 R$-ACPD isomer that is present in a $1200 \mathrm{nmol}$ dose of trans-ACPD.

The lack of $( \pm)$ trans-AC.PD injury in cultured neurons (Koh et al., 1991) may reflect the lack of functional or critical masses of interneuronal connections that are present in situ. Furthermore, a family of metabotropic receptors have been cloned (Houamed et al., 1991; Masu et al., 1991; Tanabe et al., 1992) which are coupled to multiple transduction mechanisms including phosphoinositide hydrolysis, stimulation of cAMP formation, inhibition of cAMP formation, and the release of arachidonic acid. $( \pm)$ Trans-ACPD activates the subtypes of metabotropic glutamate receptors coupled to all of these transduction mechanisms. Therefore, it is possible that specific metabotropic glutamate-receptor effects, such as the neuronal injury demonstrated in our study, are linked to a specific metabotropic glutamate receptor subtype. This heterogeneity may explain why ccrtain tissues or in vitro preparations do not appear to express metabotropic glutamate receptor-mediated neuronal injury. The lack of compounds selective for the metabotropic glutamate receptor subtypes makes it difficult to determine the contribution of each metabotropic glutamate receptor subtype to $1 S, 3 R$ ACPD-mediated brain injury in vivo.

In addition to the direct toxicity of $1 S, 3 R$-ACPD shown here, a lower subtoxic dose of $1 S, 3 R$-ACPD (250 nmol) selectively potentiated NMDA-mediated (65\% enhancement) but not AMPA-mediated brain injury when injected intrastriatally in neonatal rats (McDonald and Schoepp, 1992). Like direct $1 S, 3 R$ ACPD toxicity, this effect was stereoselective since $1 R, 3 S$-ACPD did not potentiate NMDA toxicity. Thus, mechanisms also exist for a synergistic interaction between $1 S, 3 R$-ACPD-sensitive metabotropic receptors and NMDA receptors, and these mechanisms play a prominent role in neonatal brain injury. It has been shown that depolarizing responses to NMDA, but not AMPA, are selectively potentiated by $1 S, 3 R$-ACPD in area CA 1 of rat hippocampus (Aniksztein et al., 1991; Harvey et al., 1991). Thus, potentiation of NMDA responses may have important implications for synaptic plasticity and neuropathology of acute and chronic neurologic disorders. However, in this study relatively higher doses of $1 S, 3 R$-ACPD induced brain injury that was not altered by concurrent treatment with the selective ionotropic antagonists MK-801 or GYKI-52466. Direct $1 S, 3 R$ ACPD injury and convulsions following systemic $1 S, 3 R$-ACPD were blocked by dantrolene, which has been found to antagonize intracellular calcium mobilization in various neuronal and non-

\footnotetext{
Figure 3. Histologic neuronal injury induced by $1 S, 3 R$-ACPD after injection into neonatal rat striatum. $A$, Vehicle-injected control rat. Neurons are morphologically normal. $B, 1 S, 3 R$-ACPD-injected rat. Selectively injured neurons have swollen perinuclear cytoplasm containing membranous debris and slightly shrunken, pale nuclei (arrows). Epoxy embedding, toluidine blue stain. Magnification, $320 \times$.

Figure 4. Histologic neuronal injury induced by $1 S, 3 R$-ACPD after injection into neonatal rat hippocampus. $A$, Vehicle-injected control rat. Hippocampus is morphologically normal. $B, 1 S, 3 R$-ACPD-injected rat. Injured neurons with swollen cytoplasm (arrows) appear throughout much of the hippocampus, especially in the polymorphic $(P O)$ and molecular $(M)$ layer. $C$, Detail of normal control hippocampus. $D$, Detail of swollen cytoplasm and pale nuclei characteristic of $1 S, 3 R$-ACPD-induced neuronal injury (arrows). Note the relatively few injured cells in the pyramidal cell layer $(P Y)$. $A$ and $B$, paraffin embedding, HE stain; $C$ and $D$, epoxy embedding, toluidine blue stain. Magnification: $A$ and $B, 80 \times ; C$ and $D$, $320 \times$.
} 
A

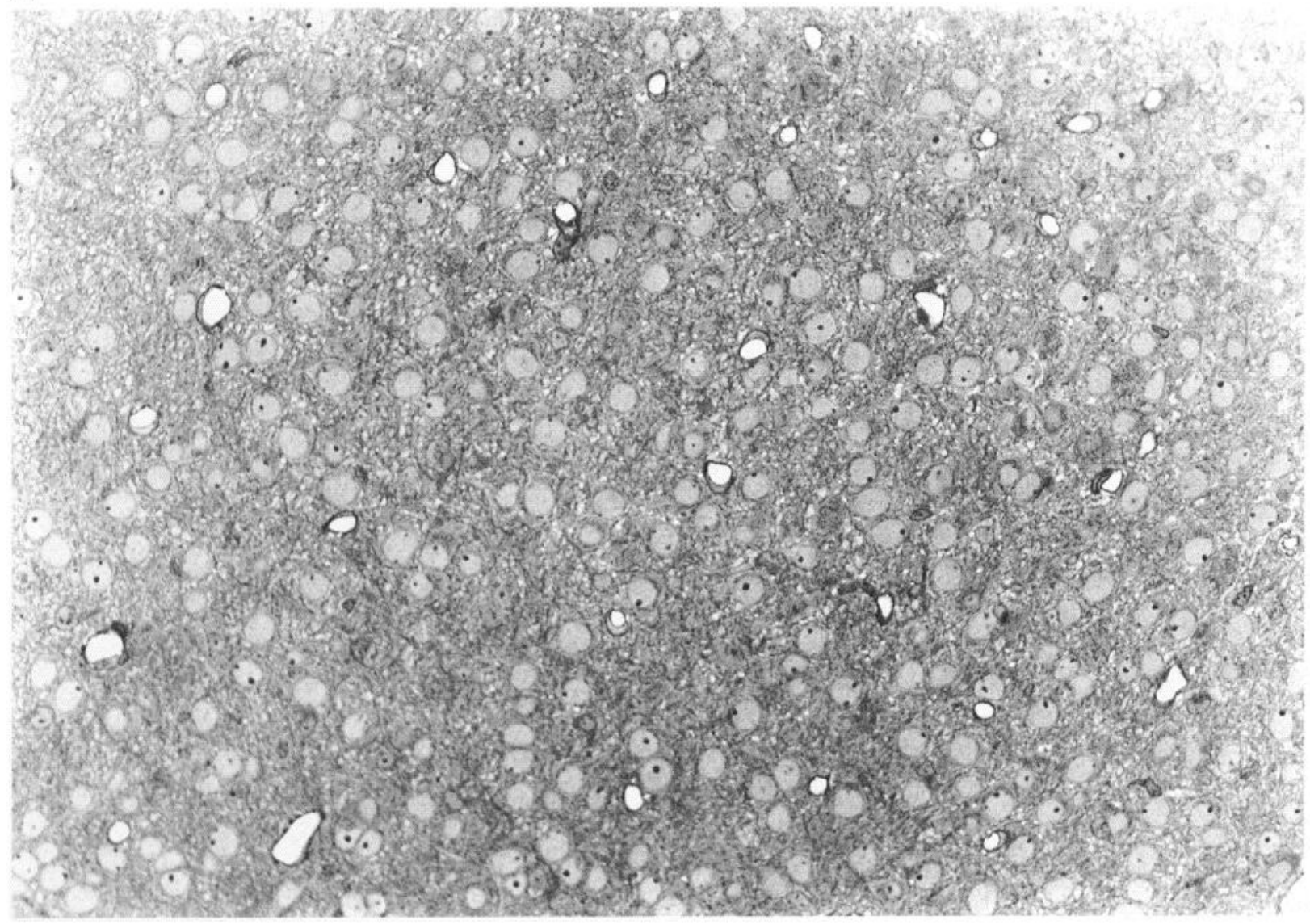

\section{B} 3.5
3

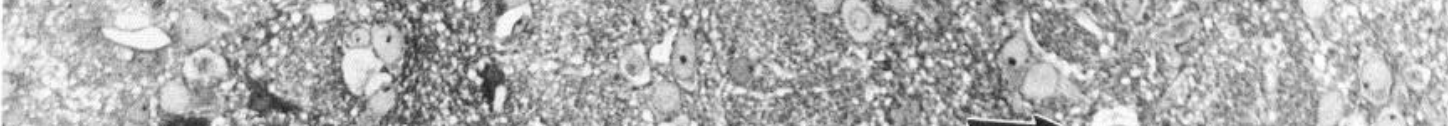

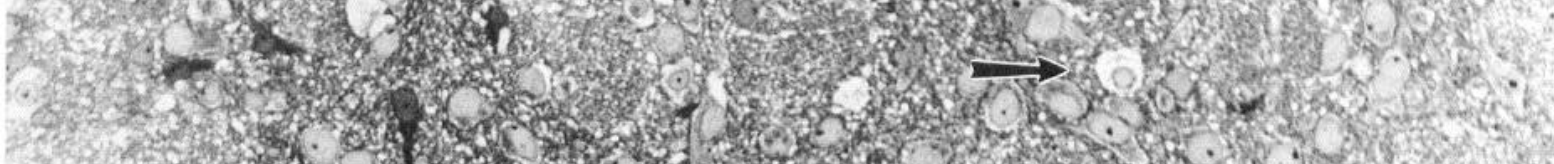

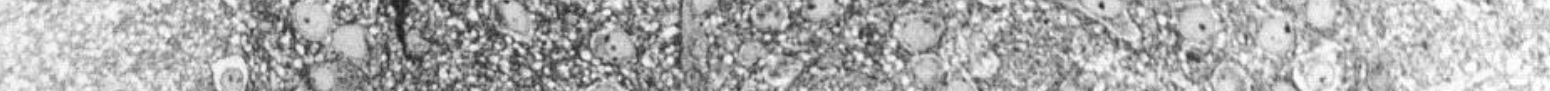

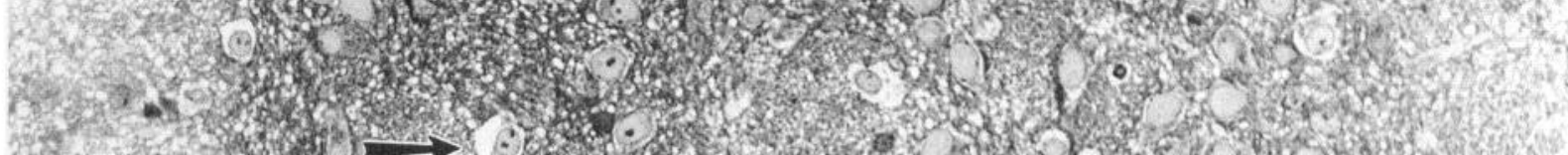

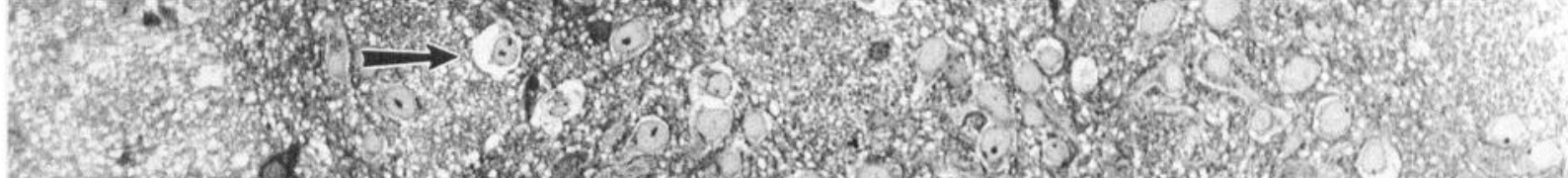

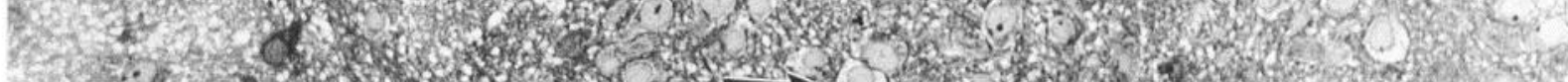

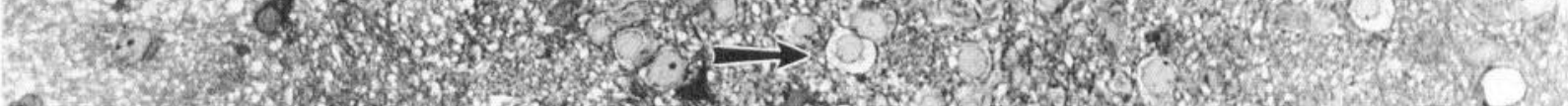

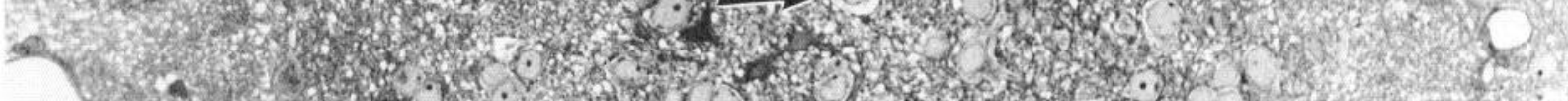

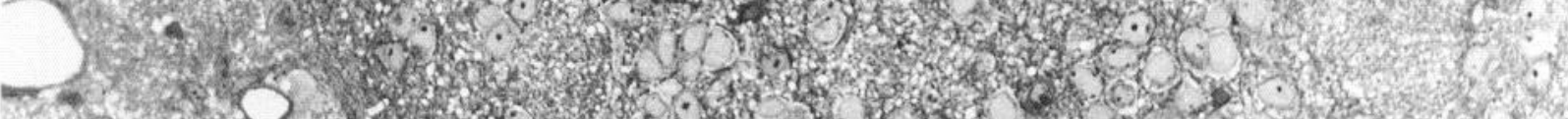

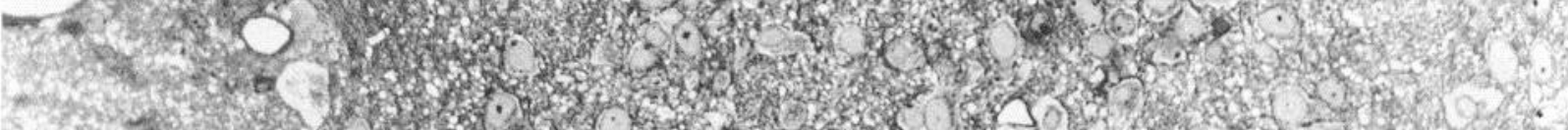

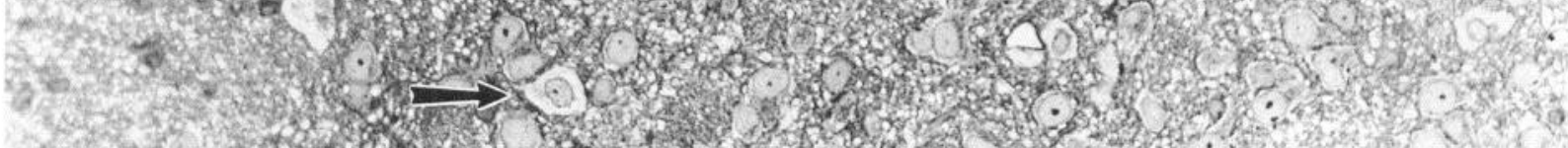

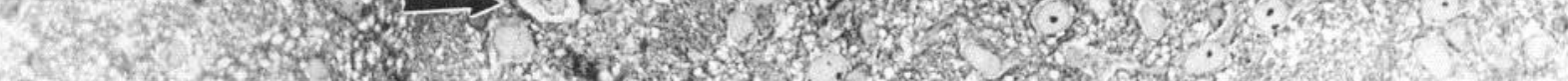

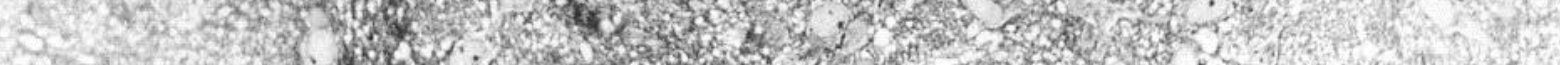
20. 







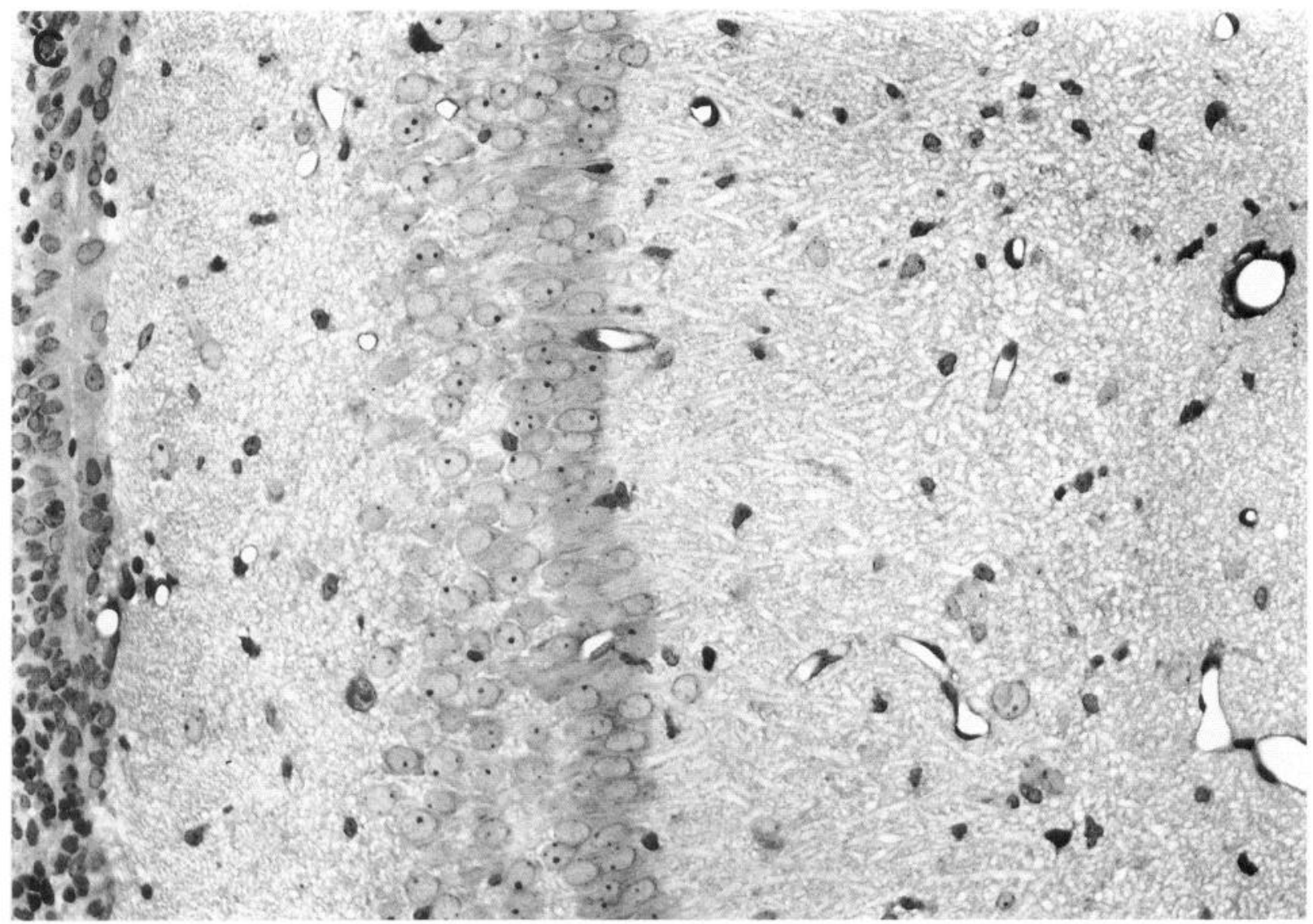

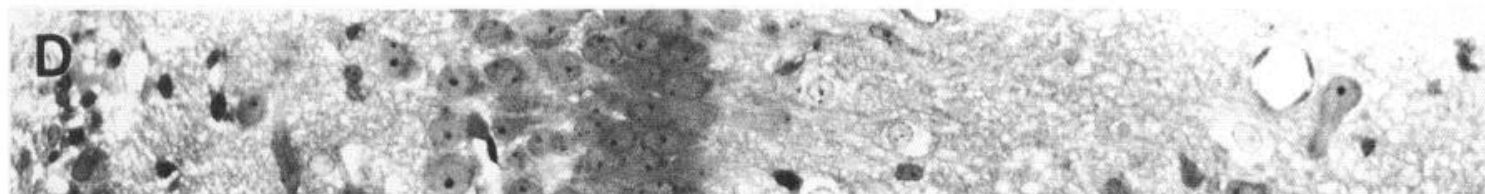

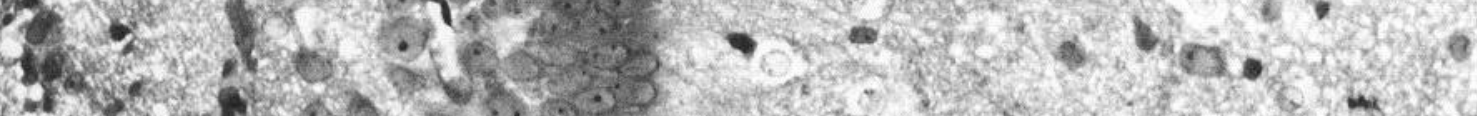

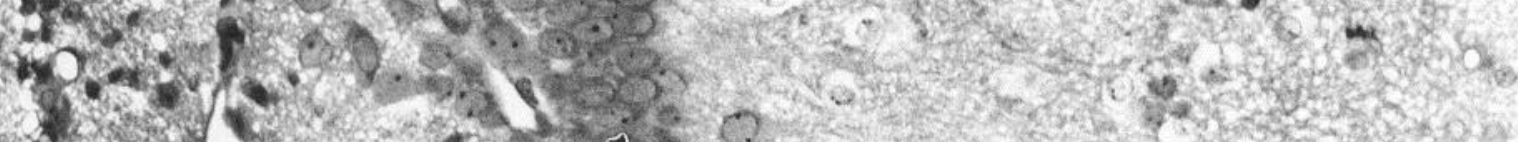

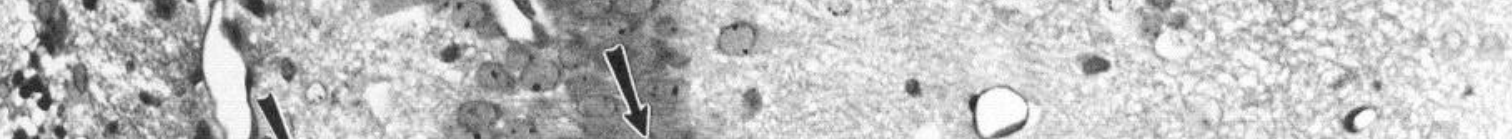

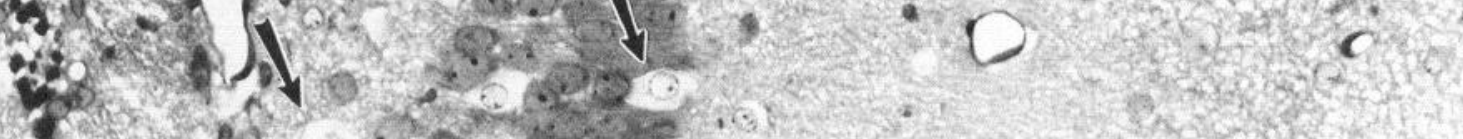

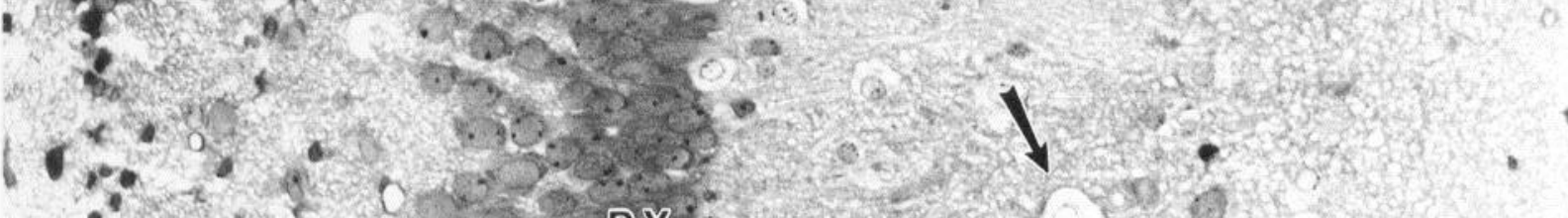

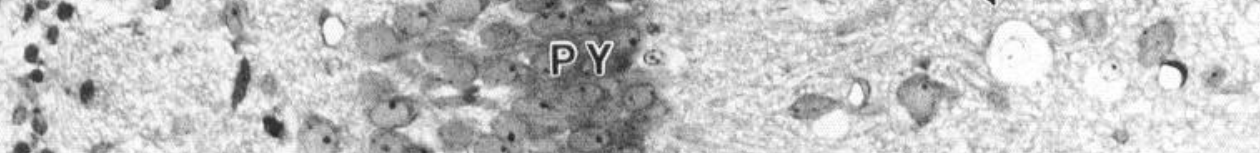

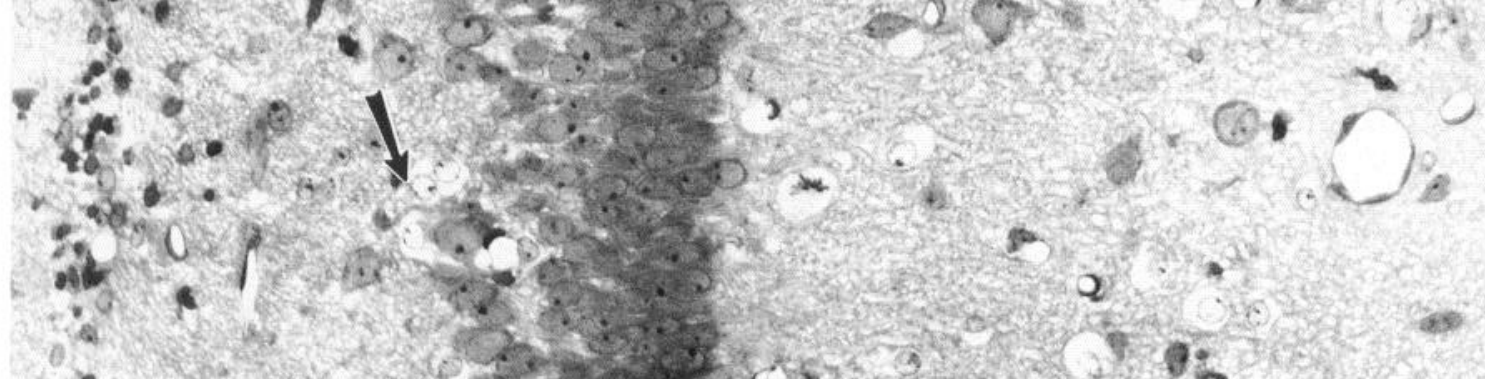

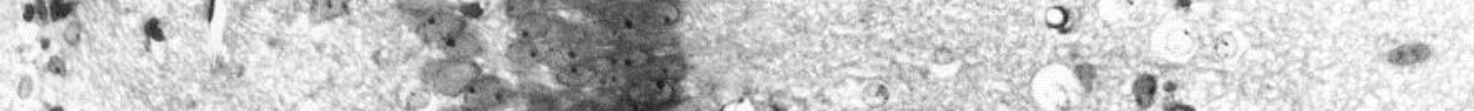




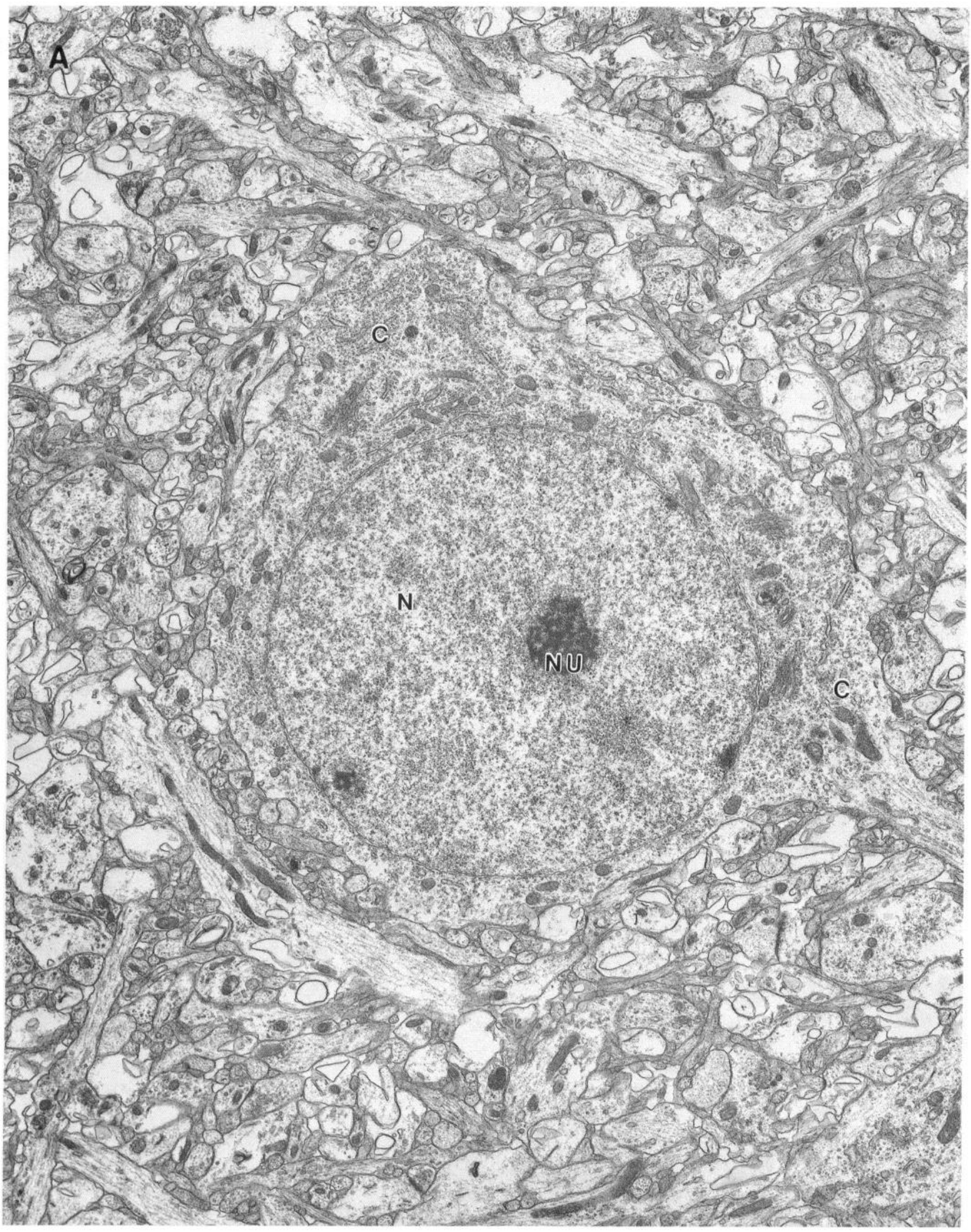

Figure 5. Transmission electron microscopic neuronal injury induced by $1 S, 3 R$-ACPD after injection into neonatal rat striatum. $A$, Vehicleinjected control rat. Neuron is morphologically normal. Note cytoplasm $(C)$, nucleus $(N)$, and nucleolus $(N U), B, 1 S, 3 R$-ACPD-injected rat. Note swollen, electron-lucent cytoplasm containing remnant mitochondria $(M)$, membranous debris $(D)$, plasmalemmal blebs $(B)$, and clear areas $(C)$. 


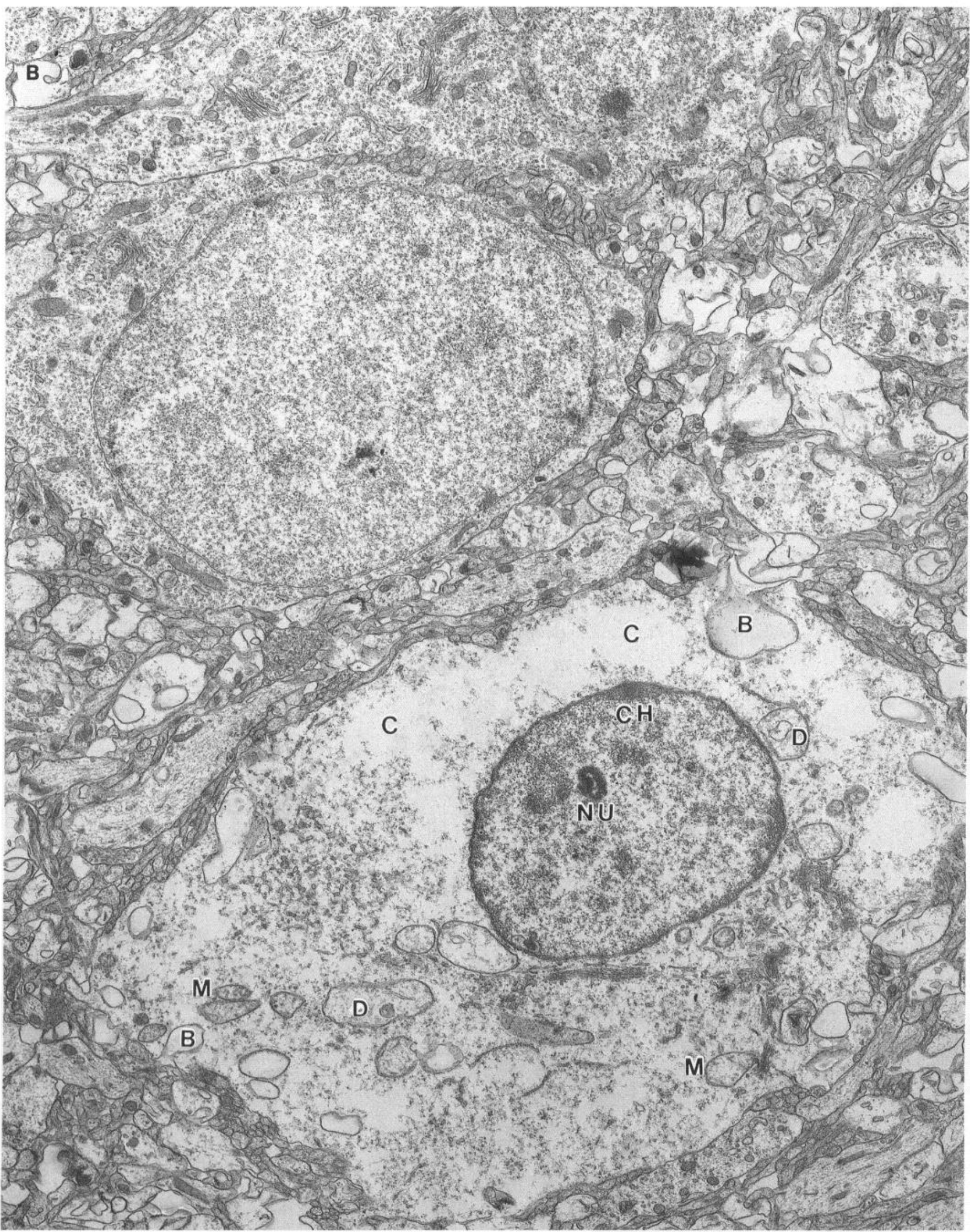

The nucleus has marginated chromatin $(\mathrm{CH})$ and an abnormal nucleolus $(\mathrm{NU})$. Adjacent neurons are morphologically normal. Magnification, $12,000 \times$ 


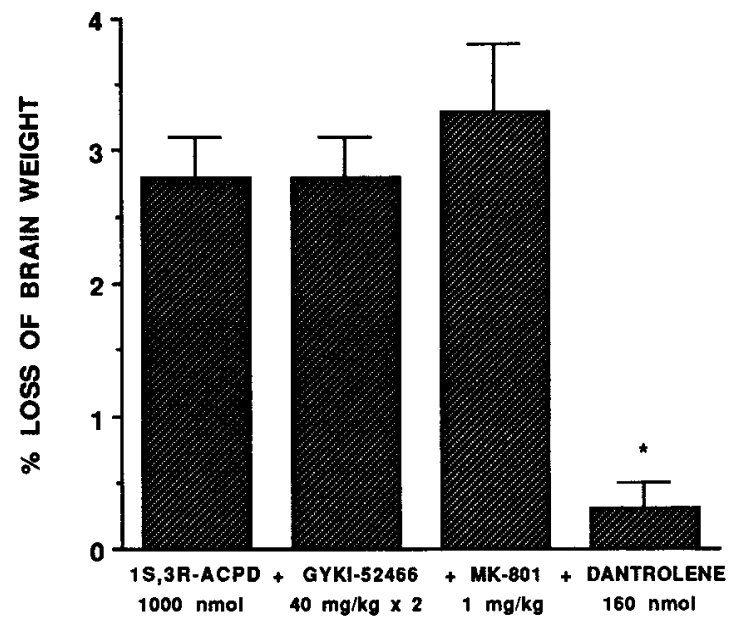

Figure 6. Comparison of the effect of selected potential neuroprotective compounds on $1 S, 3 R$-ACPD-induced brain injury in neonatal rats. PND 7 rats received unilateral intrastriatal injections of $1000 \mathrm{nmol}$ of $1 S, 3 R$-ACPD and were simultaneously treated with various compounds. Eight to 13 animals were tested in each group. GYKI-52466 $(40 \mathrm{mg} / \mathrm{kg}$, divided into two doses, concurrent and $1 \mathrm{hr}$ later) and MK$801(1 \mathrm{mg} / \mathrm{kg}$ ) were administered intraperitoneally (in $0.05 \mathrm{ml} \mathrm{PBS}, \mathrm{pH}$ 7.4). Dantrolene $(160 \mathrm{nmol})$ was coinjected with $1 S, 3 R$-ACPD (1000

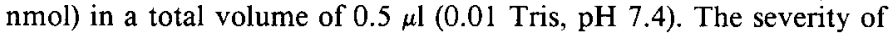
brain injury was evaluated on PND 12 by comparison of hemisphere weight disparities as detailed for Figure $3 . *, p<0.001,1 S, 3 R$-ACPD group versus $1 S, 3 R$-ACPD plus dantrolene group.

neuronal cell types (Ward et al., 1986). In cultured cerebral cortical neurons it has recently been reported that neuronal injury can be induced by the metabotropic glutamate agonist quisqualate. Although quisqualate also activates AMPA receptors, this neurotoxic effect of quisqualate in cultured neurons is not blocked by AMPA antagonists, but can also be attenuated by dantrolene (Frandsen and Schousboe, 1992). The ability of dantrolene to inhibit $1 S, 3 R$-ACPD seizures and $1 S, 3 R-A C P D-$ induced brain injury suggests that these effects are linked to intracellular calcium mobilization, likely via a phosphoinositide-coupled metabotropic glutamate receptor subtype(s). Metabotropic-stimulated phosphoinositide hydrolysis is markedly enhanced during the neonatal period, with maximal activity occurring at PND 7 in rats. (Nicoletti et al., 1986b; Schoepp and Hillman, 1990; for review, see McDonald and Johnston, 1990). Thus, this metabotropic glutamate receptor-mediated calcium mobilization also may play a role in pathophysiology of excitatory amino acids during early postnatal development.

Ultimately, the discovery of metabotropic receptor subtypespecific agonists and antagonists will provide the tools for further delineation of the pathophysiologic roles of metabotropic receptor subtypes in seizures and brain injury. The ability of $1 S, 3 R$ ACPD to produce seizures, direct neurotoxicity (at higher doses), and enhancement of NMDA-mediated brain injury (at lower doses) in PND 7 rats may provide useful systems for characterizing the pathophysiologic roles of specific metabotropic glutamate receptors.

\section{References}

Aniksztein L, Bregestovski P, Ben-Ari Y (1991) Selective activation of quisqualate metabotropic receptor potentiates NMDA but not AMPA responses. Eur J Pharmacol 205:327-328.

Chou TC, Talalay P (1983) Analysis of combined drug effects: a new look at a very old problem. Trends Pharmacol Sci 11:450-454.
Desai MA, Conn PJ (1990) Selective activation of phosphoinositide hydrolysis by a rigid analogue of glutamate. Neurosci Lett 109:157162.

Frandsen A, Schousboe A (1992) Mobilization of dantrolene-sensitive intracellular calcium pools is involved in the cytotoxicity induced by quisqualate and $N$-methyl-D-aspartate but not by 2-amino-3-(3-hydroxy-5-mcthylisoxazol-4-yl)propionate and kainate in cultured cerebral cortical neurons. Proc Natl Acad Sci USA 89:2590-2594.

Harvey J, Frenguelli BG, Sunter DC, Watkins JC, Collingridge GL (1991) The actions of $1 S, 3 R-A C P D$, a glutamate metabotropic receptor agonist, in the area CA1 of the rat hippocampus. Br J Pharmacol 104:75.

Houamed KM, Kuijper JL, Gilbert TL, Haldeman BA, O'Hara PJ, Mulvihill ER, Almers W, Hagen FS (1991) Cloning, expression, and gene structure of a $G$ protein-coupled glutamate receptor from rat brain. Science 252:1318-1321.

Ikonomidou C, Mosinger JL, Shahid Salles K, Labruyere J, Olney JW (1989) Sensitivity of the developing brain to hypobaric/ischemic damage parallels sensitivity to $N$-methyl-D-aspartate neurotoxicity. J Neurosci 9:2809-2818.

Irving AJ, Schofield JG, Watkins JC, Sunter DC, Collingridge GL (1990) $1 S, 3 R$-ACPD stimulates and $\mathrm{L}$-AP3 blocks $\mathrm{Ca}^{++}$mobilization in rat cerebellar neurons. Eur J Pharmacol 186:363-365.

Koh J-Y, Palmer E, Lin A, Cotman CW (1991) A metabotropic glutamate receptor agonist does not mediate neuronal degeneration in cortical culture. Brain Res 561:338-343.

Masu M, Tanabe MK, Tsuchida K, Shigemoto K, Nakanishi S (1991) Sequence and expression of a metabotropic glutamate receptor. $\mathrm{Na}$ ture 349:760-765.

McDonald JW, Johnston MV (1990) Physiological and pathophysiological roles of excitatory amino acids during central nervous system development. Brain Res Rev 15:41-70.

McDonald JW, Schoepp DD (1992) The metabotropic excitatory amino acid receptor agonist $1 S, 3 R$-ACPD selectively potentiates $N$-methylD-aspartate-induced brain injury. Eur J Pharmacol 215:353-354.

McDonald JW, Silverstein FS, Johnson MV (1988) Neurotoxicity of $N$-methyl-D-aspartate is markedly enhanced in developing rat central nervous system. Brain Res 459:200-203.

McDonald JW, Roeser NF, Silverstein FS, Johnson MV (1989) Quantitative assessment of neuroprotection against NMDA-mediated brain injury. Exp Neurol 106:289-296.

Monaghan DT, Bridges RJ, Cotman CW (1989) The excitatory amino acid receptors: their classes, pharmacology, and distinct properties in the function of the central nervous system. Annu Rev Pharmacol Toxicol 29:365-402.

Nicoletti F, Iadarola MJ, Chaung DM, Roth BL, Costa E (1986a) Coupling of inositol phospholipid metabolism with excitatory amino acid recognition sites in rat hippocampus. J Neurochem 46:40-46.

Nicoletti F, Iadarola MJ, Wroblewski JT, Cosla E (1986b) Excitatory amino acid recognition sites coupled with inositol phospholipid metabolism: developmental changes and interaction with alpha-1-adrenoreceptors. Proc Natl Acad Sci USA 83:1931-1935.

Olney JW (1971) Glutamate-induced neuronal necrosis in the infant mouse hypothalamus. J Neuropathol Exp Neurol 30:75-90.

Olney JW (1978) Neurotoxicity of excitatory amino acids. In: Kainic acid as a tool in neurobiology (McGeer EG, Olney JW, McGeer TL, eds), pp 95-121. New York: Raven.

Palmer E, Monaghan DT, Cotman CW (1989) Trans-ACPD, a selective agonist to the PI-coupled excitatory amino acid receptor. Eur J Pharmacol 166:585-587.

Pearce B, Albrecht J, Morrow C, Murphy S (1986) Astrocyte glutamate receptor activation promotes inositol phospholipid turnover and calcium influx. Neurosci Lett 72:335-340.

Sacaan AI, Schoepp DD (1992) Activation of hippocampal metabotropic excitatory amino acid receptors leads to seizures and neuronal damage. Neurosci Lett 139:77-82.

Schoepp DD, Hillman CC (1990) Developmental and pharmacological characterization of quisqualate, ibotenate, and trans-1-amino1,3-cyclopentanedicarboxylic acid stimulations of phosphoinositide hydrolysis in rat cortical brain slices. Biogenic Amines 7:331-340.

Schoepp DD, Johnson BG (1988) Excitatory amino acid agonistantagonist interactions at 2-amino-4-phosphonobutyric acid-sensitive quisqualate receptors coupled to phosphoinositide hydrolysis in slices of the rat hippocampus. J Neurochem 50:1605-1613.

Schoepp D, Bockaert J, Sladeczek F (1990a) Pharmacological and 
functional characteristics of metabotropic excitatory amino acid receptors. Trends Pharmacol Sci 11:508-515.

Schoepp DD, Gamble AY, Salhoff CR, Johnson BG, Ornstein PL (1990b) Excitatory amino acid-induced convulsions in neonatal rats mediated by distinct receptor subtypes. Eur J Pharmacol 182:421427.

Schoepp DD, Johnson BG, Salhoff CR, McDonald JW, Johnston MV (1991a) In vitro and in vivo pharmacology of trans- and cis-ACPD: dissociation of metabotropic and ionotropic excitatory amino acid receptor'effects. J Neurochem 56:1789-1796.

Schoepp DD, Johnson BG, True RA, Monn JA (1991b) Comparison of $(1 S, 3 R)$-1-aminocyclopentane-1,3-dicarboxylic acid $(1 S, 3 R$ ACPD)- and $1 R, 3 S$-ACPD-stimulated brain phosphoinositide hydrolysis. Eur J Pharmacol [Mol Pharmacol] 207:351-353.

Schoepp DD, Johnson BG, Monn JA (1992a) Inhibition of cyclic AMP formation by a selective metabotropic glutamate antagonist. J Neurochem 58:1 184-1186.
Schoepp DD, Johnson BG, Sacaan AI, True RA, Monn JA (1992b) In vitro and in vivo pharmacology of $1 S, 3 R$ - and $1 R, 3 S$-ACPD: evidence for a role of metabotropic glutamate receptors in striatal motor function. Mol Neuropharmacol 2:33-37.

Sladeczek F, Pin JP, Recasens M, Bockaert J, Weiss S (1985) Glutamate stimulates inositol phosphate formation in striatal neurons. Nature 317:717-719.

Sugiyama HI, Ito I, Hirono C. (1987) A new type of glutamate receptor linked to inositol phospholipid metabolism. Nature 325:531-533.

Tanabe Y, Masu M, Ishii T, Shigemoto R, Nakanishi S (1992) A family of metabotropic glutamate receptors. Neuron 8:169-179.

Ward A, Chaffman MO, Sorkin EM (1986) Dantrolene. A review of its pharmacodynamic and pharmacokinetic properties and therapeutic use in malignant hyperthermia, the neuroleptic malignant syndrome and an update of its use in muscle spasticity. Drugs 32:130168. 